\title{
CYCLIC INVARIANCE UNDER MULTI-VALUED MAPS ${ }^{1}$
}

\author{
A. D. WALlace
}

In what follows it is always assumed that $X, Y$ are compact (=bicompact) connected Hausdorff spaces each containing more than one point.

Let $f$ denote a function which assigns to each $x$ in $X$ a subset $f(x)$ of $Y$. We suppose that the sets $\{f(x)\}$ cover $Y$. By definition

$$
f^{-1}(y)=\{x \mid x \in f(y)\} .
$$

It is assumed that the sets $\left\{f^{-1}(y)\right\}$ cover $X$. The functions $f$ and $f^{-1}$ play dual roles inasmuch as $f=\left(f^{-1}\right)^{-1}$. If $f$ is single-valued, then $f^{-1}$ is the inverse of $f$ in the usual meaning of the term. For $A \subset X, B \subset Y$ we define

$$
f(A)=\bigcup\{f(x) \mid x \in A\}, \quad f^{-1}(B)=U\left\{f^{-1}(y) \mid y \in B\right\} .
$$

When $f$ is single-valued we know that continuity is equivalent to the assertion that $A, B$ closed imply $f(A), f^{-1}(B)$ closed. When $f$ is multi-valued we take this as a definition of continuity. It does not follow, as in the single-valued case, that $f^{-1}(B)$ is open if $B$ is open. These definitions include both a single-valued map (=continuous function) and its inverse.

In this note we show that certain theorems of analytic topology carry over to multi-valued maps (=continuous multi-valued functions as defined above). Some of our results are new even for singlevalued maps. Except for fixed-point theorems there seem to be no results in the literature for multi-valued maps.

We say that $f$ is anarthric if it is continuous and if for $y \in Y$ no $x \in X-f^{-1}(y)$ separates $f^{-1}(y)$ in $X$. If $f$ is single-valued and nonalternating, then $f$ is anarthric. See Wallace $[2],{ }^{2}[3]$, and [4] and Whyburn [5] and [6]. It is clear that if $f$ is the inverse of a singlevalued map, then $f$ is anarthric.

For simplicity we write $P \mid Q$ to mean that the sets $P$ and $Q$ are mutually separated. Also if $p, q \in X$, then $p \sim q$ means that no point separates $p$ and $q$ in $X$.

THEOREM 1. In order that the multi-valued map $f$ be anarthric each of the following conditions is both necessary and sufficient:

Received by the editors June 28, 1948.

1 This work was done under Contract N7-onr-434, Task Order 3, Navy Department, The Office of Naval Research.

${ }^{2}$ Numbers in brackets refer to the bibliography at the end of the paper. 
(i) If $X=M \cup N$, where $M$ and $N$ are continua meeting in a cutpoint $x$, and $K$ is any continuum meeting $M$, then $f(M \cap K)=f(M) \cap f(K)$.

(ii) If $H$ is a subcontinuum of $Y$ and $K$ is a subcontinuum of $X$ and $K \cap f^{-1}(H)=P \cup Q, P \mid Q$, then there exist points $p \in P, q \in Q$ such that $p \sim q$.

Proof. We show that (i) holds if $f$ is anarthric. Now the conclusion follows at once if $K$ is disjoint with either $M-x$ or $N-x$. We assume therefore that $K$ meets both of these sets and that $y \in f(K) \cap f(M)$ $-f(K \cap M)$, the inclusion $f(M \cap K) \subset f(K) \cap f(M)$ clearly holding. Then $f^{-1}(y)$ meets both $K$ and $M$ but not $K \cap M$. Hence $x$ is not in $f^{-1}(y)$ since $x \in K \cap M$. But $f^{-1}(y)$ meets both $M-x$ and $N-x$, a contradiction.

Next, (i) implies (ii). For let $K_{0}$ be a continuum contained in $K$ and irreducible between the disjoint closed sets $P$ and $Q$. Let $p \in P$ $\cap K_{0}, q \in Q \cap K_{0}$ and suppose that $x$ separates $p$ and $q$ in $X$. Then $p \cup q \cup\left(K_{0}-(P \cup Q)\right)$ is connected and so contains $x$. Thus $x$ is not in $f^{-1}(H)$ and so $f(x)$ does not intersect $H$. We have a decomposition $X=M \cup N$ with $M$ and $N$ closed, $M \cap N=x$ and $p \in M, q \in N$. Now $f(x)=f(M) \cap f(N)$ as we see by taking the $K$ of (i) to be the present $N$. But $H=(H \cap f(M)) \cup(H \cap f(N))$ and so $H \cap f(M) \cap f(N)$ is not void since $H$ is a continuum contrary to the fact that this intersection is $H \cap f(x)$.

Finally (ii) implies that $f$ is anarthric by taking $H=y$ and $K=X$.

We remark that it is sufficient to take $K=N$ in (i) and in (ii) to take $K=X$.

We recall briefly some definitions and results mostly contained in Wallace [2]. These reduce to the well known cyclic element theory if $X, Y$ are metric and locally connected. See Whyburn [5].

By an $A$-set we mean a closed set $H$ such that if $z \in X-H$, then $X=M \cup N$ with $M \cap N=x,(M-x) \mid(N-x), H \subset M, z \in N-x$. It is easily seen that an $A$-set is a chain (Wallace [2]) and hence a continuum, that the intersection of any collection of $A$-sets is again an $A$-set and that the union of two intersecting $A$-sets is also an $A$-set.

A prime-chain is a chain which is either an end point, a cutpoint or a nondegenerate minimal chain. One can replace "chain" by " $A$-set" in the last sentence. It is readily seen that if a chain $A$ is met by a prime-chain $E$ in two points or in a non-cutpoint, then $E \subset A$.

A nodal set is a closed set which meets the closure of its complement in a single point. It is readily seen that an $A$-set is the intersection of all the nodal sets containing it and (since each nodal set is an $A$-set) that any intersection of nodal sets is an $A$-set. 
THEOREM 2. In order that the multi-valued map $f$ be anarthric it is necessary and sufficient that for any $A$-set $H$ in $X$ and any subcontinuum $K$ of $X$ meeting $H$ we have $f(H \cap K)=f(H) \cap f(K)$.

Proof. Suppose that $f$ is anarthric. It is enough to show that, for any $y$ in $Y, f^{-1}(y) \cap H, f^{-1}(y) \cap K$ nonvoid imply $f^{-1}(y) \cap H \cap K$ nonvoid. Now $H \cup K$ is a continuum and so by (ii) there exist points $p \in H \cap f^{-1}(y), q \in K \cap f^{-1}(y)$ with $p \sim q$, assuming of course that our implication is not valid. Let $E$ be the prime-chain containing $p \cup q$ (Wallace [2]). Then $E \cap H$ nonvoid implies that $E \cup H$ is an $A$-set. Thus, since $K$ is a continuum, we know that $K \cap(E \cup K)=(K \cap E)$ $\cup(K \cap H)$ is connected and so $E \cap H \cap K$ is not void. Hence $E$ must contain two distinct points of $H$ since $p \in f^{-1}(y)$ and this latter set does not meet $H \cap K$ by assumption. From this it follows that $E \subset H$ and so $q \in H$, a contradiction.

The sufficiency is readily inferred from the remarks following Theorem 1. The result fails unless it is required that $H$ and $K$ meet. For if $X$ is the union of the unit circle and the segment from $(1,0)$ to $(2,0)$ and $Y$ is the unit circle and $f$ is the map $X$ onto $Y$ carrying the segment into $(1,0)$, then taking $Y=H$ and $K=(2,0)$ we see that the conclusion fails.

THEOREM 3. In order that the multi-valued map $f$ be anarthric it is necessary and sufficient that if $\{A\}$ is any collection of $A$-sets with the finite intersection property, then $f(\cap A)=\cap f(A)$.

Proof. If $f$ is anarthric it is sufficient to prove that, if $y \in Y$, the proposition " $f^{-1}(y)$ meets every set in $\{A\}$ " implies " $f^{-1}(y)$ meets $\cap A$." To this end show that $\left\{f^{-1}(y) \cap A\right\}$ has the finite intersection property. Or, for any $A_{1}, A_{2}, \cdots, A_{n}$ in $\{A\}$ we have $f^{-1}(y)$ $\cap A_{1} \cap \cdots \cap A_{n}$ nonvoid. Now by Theorem 2 we see that $f\left(A_{1} \cap \cdots \cap A_{n}\right)=f\left(A_{1}\right) \cap \cdots \cap f\left(A_{n}\right)$. Thus if $f^{-1}(y)$ intersects every $A_{i}$, then $f^{-1}(y)$ also intersects $A_{1} \cap \cdots \cap A_{n}$.

The sufficiency follows from the fact that, in Theorem 2 , it is enough to take $K$ an $A$-set.

THEOREM 4. If $f$ is anarthric and the image of each cutpoint is a point, then the image of a nodal set is a nodal set.

This follows without difficulty from (i) of Theorem 1 . The result is false if the condition, that the image of a cutpoint be a point, is deleted. In the $(u, v)$ plane let $Y$ be the circle $u^{2}+v^{2}=4$ and $X$ the union of the circles $(u+1)^{2}+v^{2}=1,(u-1)^{2}+v^{2}=1$. Define $g(u, v)$ $=\left(u,\left(2 u-u^{2}\right)^{1 / 2}\right)$ if $v$ is non-negative and $g(u, v)=\left(u,-\left(2 u-u^{2}\right)^{1 / 2}\right)$ if $v$ is nonpositive. Let $f=g^{-1}$; then $f$ is anarthric but the left-hand 
circle is mapped by $f$ into the left-hand semicircle of $Y$, which is not a nodal set.

THEOREM 5. If $f$ is anarthric and the image of a cutpoint is a point, then the image of an $A$-set is an A-set or a point.

Proof. If $H$ is an $A$-set, then $H$ is the intersection of all the nodal sets $\{N\}$ which contain it. By Theorems 3 and 4 we have $f(H)=\cap f(N)$. But each $f(N)$ is a nodal set and thus an $A$-set. Then $f(H)$ is an $A$-set since it is an intersection of $A$-sets.

In the case in which $f$ is non-alternating and $X$ is a Peano space this result is due to G. E. Schweigert.

Let us denote, for any non-null set $A$, the intersection of all the $A$-sets which contain $A$ by $C(A)$. It then follows from Theorem 5 (see the proof of (3.14) in Wallace [2]) that we have the following corollaries.

Corollary. For any nonempty set $A \subset X, C(f(A)) \subset f(C(A))$.

CoRollary. Let $f$ be a single-valued map of $X$ onto $Y$ such that $f^{-1}(y)$ is a point for each cutpoint $y$ in $Y$. Then the inverse of an $A$-set is an $A$-set or a point.

According to Kelley [1] a central set is an intersection of a finite number of nodal sets. From Theorems 3 and 4 we have the following theorem.

THEOREM 6. Iff is anarthric and the image of each cutpoint is a point, then the image of a central set is a central set.

TheOREM 7. In order that a multi-valued map be anarthric it is necessary and sufficient that no $A$-set separate the inverse of a point.

Proof. The condition is clearly sufficient since each cutpoint is an $A$-set. Suppose that some $A$-set $A$ separates the inverse of a point so that we have $X-A=U \cup V, U \mid V$, with $f^{-1}(y)$ meeting both $U$ and $V$ but not $A$. Now $A \cup U$ and $A \cup V$ are $A$-sets, say $H$ and $K$. Then $f^{-1}(y)$ intersects both $H$ and $K$ but not $H \cap K$ contrary to the fact $f(H \cap K)=f(H) \cap f(K)$,

Our next result generalizes a noteworthy theorem of G. T. Whyburn [5].

THEOREM 8. Let $f$ be an anarthric map such that the image of a cutpoint is a point and let $E$ be a prime-chain in $Y$. Then there is a primechain $F$ in $X$ such that $E \subset f(F)$. If $F^{\prime}$ is any other prime-chain in $X$, then $f\left(F^{\prime}\right)$ meets $E$ in at most one point. 
Proof. We may suppose that $E$ is nondegenerate. Using the Hausdorff maximality principal (Zorn's lemma) we see that there exists a collection $\{A\}$ of $A$-sets maximal relative to the properties that (i) $E \subset f(A)$ and (ii) no finite collection of $A$ 's has a void intersection. Let $F$ be the intersection of these $A$ 's so that, since $f(F)=\cap f(A)$, we know that $E$ is a subset of $f(F)$. Now if $F$ is a point it is either an end point or a cutpoint and so a prime-chain. Suppose that $F$ contains more than one point and is not a prime-chain. Then $X=M \cup N$ with $M \cap N=x,(M-x) \mid(N-x)$ with $F$ meeting each of these separands. We conclude that $Y=f(M) \cup f(N), f(x)=y=f(M) \cap f(N)$. Now if $E$ is contained in $f(M)$, then also $E \subset f(M) \cap f(F)=f(M \cap F)$. Since $M$ and $F$ are $A$-sets so also is $M \cap F$ and this latter is a proper subset of $F$ contrary to the maximality of $\{A\}$. Accordingly we conclude that $E$ meets both $f(M)-y$ and $f(N)-y$, an impossibility since $E$ is a prime-chain.

Let $F^{\prime}$ be a prime-chain distinct from $F$ such that $f\left(F^{\prime}\right)$ contains two points of $E$. Then $X=M \cup M^{\prime}$ where $M, M^{\prime}$ are closed, intersect in a cutpoint $x$ and $F \subset M, F^{\prime} \subset M^{\prime}$. Then $f(x)=y \in Y$. As before $Y=f(M) \cup f\left(M^{\prime}\right)$. If $f\left(M^{\prime}\right) \subset f(M)$, then $f\left(M^{\prime}\right)=y$ contrary to the fact that $f\left(F^{\prime}\right) \subset f\left(M^{\prime}\right)$ and contains two points. It then follows that, as above, the point $y$ cuts $E$ in $X$, a contradiction.

\section{BIBLIOGRAPHY}

1. J. L. Kelley, Simple links and fixed sets under continuous mappings, Amer. J. Math. vol. 69 (1947) p. 349.

2. A. D. Wallace, Monotone transformations, Duke. Math. J. vol. 9 (1942) pp. 487-506.

3. - On non-alternating transformations, Bull. Amer. Math. Soc. vol. 49 (1943) p. 62.

4. - A structural property of transformations, Bull. Amer. Math. Soc. vol. 49 (1943) p. 699.

5. G. T. Whyburn, Analytic topology, Amer. Math. Soc. Colloquium Publications, vol. 28, 1942.

6. - Uniqueness of the inverse of a transformation, Duke Math. J. vol. 12 (1945) pp. 317-323.

The TUlane University 